\title{
A progress report on the study of the optical variability of the old nova HR Del
}

\author{
I. Voloshina, ${ }^{1}$ M. Friedjung, ${ }^{2}$ M. Dennefeld ${ }^{2}$ and V. Sementsov ${ }^{1}$ \\ ${ }^{1}$ Sternberg Astronomical Institute, Moscow State University, Moscow, Russia \\ ${ }^{2}$ Institut d'Astrophysique de Paris, CNRS, Paris, France
}

\begin{abstract}
The cataclysmic variable and old nova HR Del has been studied using ground-based photometry and spectroscopy over a period of more than 6 years. Some long term variations have been found, but no clear correlation established up to now between photometric, and spectroscopic changes.
\end{abstract}

Individual Objects: Old nova HR Del

With the aim of finding more indications of continuing activity of the old nova HR Del, new spectral and photometric observations have been obtained between 2002 and 2008, with however an irregular time sampling. Results from a preliminary study were presented by Friedjung et al. (2005). Here we present mainly the results of our complete photometric observations of this object.

HR Del (or Nova Del 1967) was a bright classical nova with however unusual properties. It brightened in July 1967 to a magnitude near $5^{\mathrm{m}} .5$ from pre-outburst magnitude of about $12^{\mathrm{m}}$. After remaining 5 months near this magnitude, it brightened to a maximum $m_{v} \sim 3.5$, followed by an extremely slow irregular decline. The unusual nature of this nova might be due to the presence of a wind which was optically thin in the continuum before maximum, and whose velocity decreased during that stage, unlike that of the optically thick winds normally seen after maximum of classical novae (Friedjung 1992). The mass of the white dwarf component is low $-(0.55 \div 0.75) \mathrm{M}_{\odot}-$, while the orbital period is 0.214165 (Kürster \& Barwig 1988). HR Del was unusually bright both before and long after its outburst (Selvelli \& Friedjung 2003).

Two kinds of photometric observations were made:

- To construct the overall light curve, daily estimates of HR Del's magnitude were made with the UBV photometer on the 60-cm telescope of the Sternberg Astronomical Institute in Crimea (time resolution $10 \mathrm{~s}$ ). They covered the years 2001-2006, with more than 1000 measurements in 3 bands.

- To study the short-term variability of HR Del, observations with an Ap47 CCD detector on the same telescope were obtained in 2005-2006, covering 23 nights, and containing more than 3500 measurements, with a time resolution of $30 \mathrm{~s}$ in the $\mathrm{V}$ band.

- High-resolution spectroscopic observations of the region of the $\mathrm{H} \alpha$ line were obtained with the 152-cm telescope of the Haute Provence Observatory. 


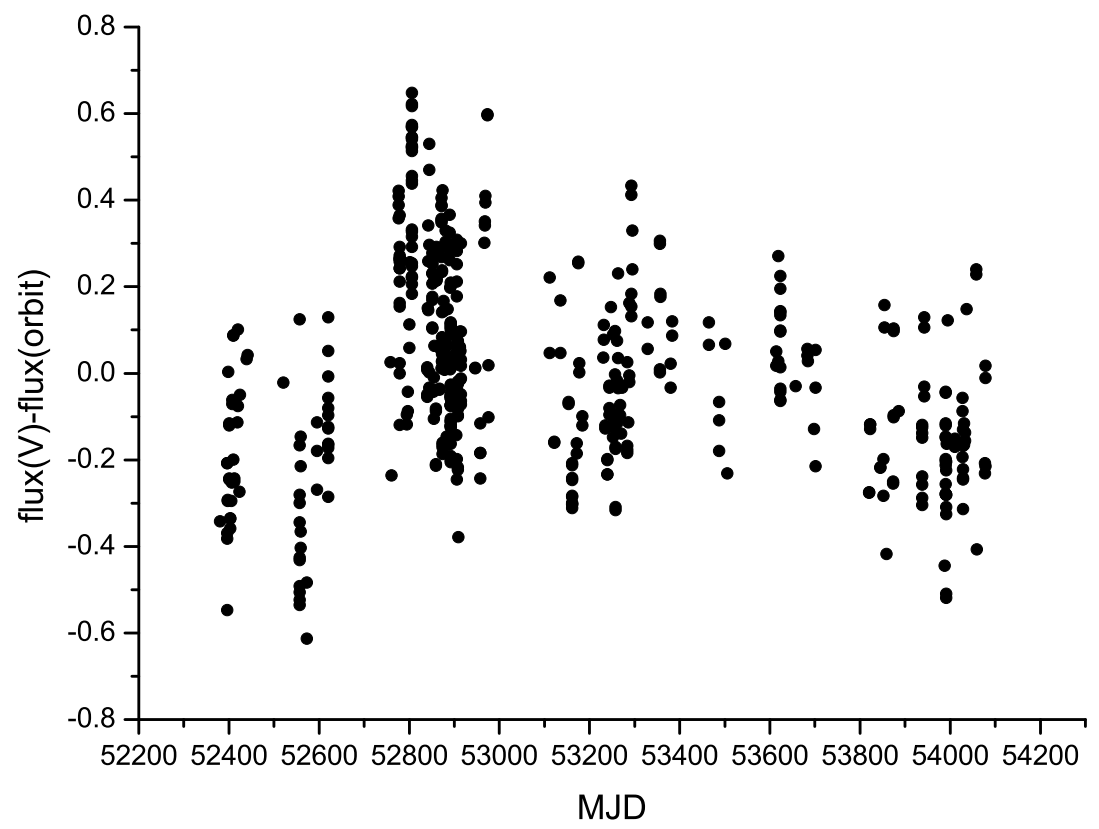

Figure 1: Long time scale flux variation of HR Del: flux in the $\mathrm{V}$ band corrected for orbital variations versus time in Modified Julian Day.

The overall light curve of HR Del was obtained from photometric observations in the $\mathrm{V}$ band and is shown in Fig. 1 after correction for orbital variations. This figure shows that, after subtraction of the orbital variations, long time scale variations exist in the light curve of HR Del. While there is evidence for long time scale variations, no short term periodic oscillations were found in the light curves determined from the CCD observations, except the orbital ones (with an amplitude up to $0^{m} .5$ ). The profiles of $\mathrm{H} \alpha$ show both a strong narrow component and weak wings. No clear evidence was found for orbital variations of the equivalent width, but long time scale variations are seen with a decrease from 2002 to 2004, followed by an increase again. More details will be presented in a forthcoming paper.

Acknowledgments. I.V. acknowledges financial support provided by RFBR through grant 06-02-16411. We also thank N. Metlova for her kind help with observations.

\section{References}

Friedjung, M. 1992, A\&A, 262, 487

Friedjung, M., Bruch, A., Voloshina, I., et al. 2005, ASP Conf. Ser., 330, 437

Selvelli, P., \& Friedjung, M. 2003, A\&A, 401, 297

Kürster, M., \& Barwig, H. 1988, A\&A, 199, 201 